Copyright by the Acoustical Society of America. Smith, J. P., Johnson, B. D., \& Burdisso, R. A. (1999). A broadband passive-active sound absorption system. Journal of the Acoustical Society of America, 106(5), 2646-2652. doi: 10.1121/1.428094

\title{
A broadband passive-active sound absorption system
}

\author{
Jerome P. Smith, Brody D. Johnson, and Ricardo A. Burdisso \\ Vibration and Acoustics Laboratories, Mechanical Engineering Department, \\ Virginia Polytechnic Institute and State University, Blacksburg, Virginia 24061-0238
}

(Received 26 August 1998; accepted for publication 7 July 1999)

\begin{abstract}
In recent years, hybrid absorption systems have been implemented which achieve high sound absorption over a broad frequency range. This work is an experimental study of a broadband hybrid absorption system which is comprised of a layer of sound-absorbing material (the passive component) positioned at a distance from a movable wall (the active component) inside an impedance tube. The movable wall is used to impose desired boundary conditions in the cavity behind the passive layer, thereby increasing the absorption of the system at frequencies where the passive material is not independently effective. Both pressure-release (i.e., minimizing the pressure at the back surface of the layer) and impedance-matching (i.e., minimizing the reflected wave from the layer) boundary conditions are studied. The performance of the hybrid system for these two boundary conditions is compared for broadband disturbances over a frequency range of 100-1000 Hz. The unmodified passive system showed absorption coefficients greater than 0.7 only above 500 $\mathrm{Hz}$, while the impedance-matching condition yielded absorption coefficients of 0.8 to 1.0 over the $100-1000-\mathrm{Hz}$ range. The impedance-matching condition provided significantly better absorption than that achieved with the pressure-release condition. The sensitivity of these control approaches to system parameters is also investigated. (C) 1999 Acoustical Society of America.
\end{abstract}

[S0001-4966(99)00711-0]

PACS numbers: 43.55.Ev [PJR]

\section{INTRODUCTION}

Before discussing previous work on hybrid absorption systems, the advantages of such a system shall be discussed. Consider an entirely passive noise control approach relying on sound-absorbing materials. An increasing amount of material is required for effective noise reduction at low frequencies, making passive methods impractical for a given application below some low-frequency limit. On the other hand, an entirely active noise control approach becomes infeasible at high frequencies, due to the increased computational loads induced by the higher sample rate and longer filter lengths necessary. It is only natural, then, to combine the two approaches, utilizing a primarily passive effect at high frequencies and a primarily active effect at low frequencies, keeping each component relatively easy (inexpensive) to implement. Guicking and Lorenz ${ }^{1}$ provide one of the earliest applications of a hybrid noise absorption system, in which a porous plate is placed in an impedance tube a small distance away from a control speaker located at the end of the tube. The signal sensed by a reference microphone in front of the panel was issued to the control speaker via a complex amplification factor so as to minimize the sound pressure sensed by a second microphone directly behind the plate. This pressurerelease condition on the back surface of the panel led to nearly perfect absorption over a frequency range from 100 to $600 \mathrm{~Hz}$.

Thenail et al. ${ }^{2}$ also investigated a hybrid absorption system making use of a fiberglass absorbing layer backed by an air cavity terminated with an active surface. A pressurerelease condition on the back surface of the fiberglass was generated, resulting in increased absorptivity over a frequency range from 200 to $800 \mathrm{~Hz}$. Both numerical and ex- perimental results were given which demonstrate sensitivity of the system to both the fiberglass thickness and porosity.

Furstoss et al. ${ }^{3}$ more recently performed a theoretical study to determine the optimal impedance at the back face of a porous layer to result in no reflection at the front surface of the layer, for oblique and normal sound incidence. The optimal impedance was based on a model of the properties of the porous layer. An experimental study utilized an accelerometer placed on the control speaker in conjunction with a nearby microphone in an error-sensing strategy designed to result in the desired impedance behind the fiberglass, and results at a single frequency were shown to compare well with the model. Another experimental study where a pressure-release condition was imposed at the back of a fiberglass panel was shown to result in high absorption coefficients over the frequency range from 150 to $500 \mathrm{~Hz}$. For oblique sound incidence, the pressure-release-imposed impedance differed significantly from the theoretical lowfrequency optimal impedance condition, but still resulted in a significant increase in the sound absorption.

A theoretical study investigating the control effort and displacements required for introducing forces to the front layer of an absorptive foam material to result in increased absorption was performed by Bolton et al. ${ }^{4}$ Partially reticulated foams with high flow resistivity were shown to have good properties for low required control effort at low frequencies.

Another twist on the active absorption approach was provided by Fuller et al., ${ }^{5}$ in which adaptive polyvinylidene fluoride foam (PVDF) was experimentally evaluated for application to radiation and reflection control. By positioning the active PVDF foam against the inside of a rigid end cap of 


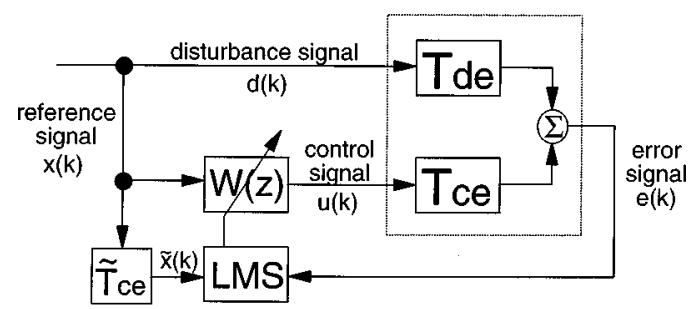

FIG. 1. Block diagram of the SISO adaptive feedforward filtered-X LMS control algorithm.

an impedance tube and minimizing the wave reflected by the foam via a wave deconvolver, an attenuation of up to $40 \mathrm{~dB}$ was achieved in the reflected wave for frequencies above 600 $\mathrm{Hz}$. The results were reported for a frequency range from 100 to $1000 \mathrm{~Hz}$. The absorption of the PVDF foam without active control was reported to be low.

Enhancing the absorption properties of passive materials, particularly in the low-frequency range, has potential applications toward improvement of anechoic facilities and in duct acoustics. Another application was demonstrated by Ver and Digman, ${ }^{6}$ who showed the application of a control technique for foam absorption enhancement in an experimentally simulated augmentor exhaust silencer tube. A pressurerelease condition introduced behind the surface of a section of resistive liner in the tube showed increases in lowfrequency noise attenuation ranging from 4 to $12 \mathrm{~dB}$ beyond the reduction obtained with the passive material only.

Beyene and Burdisso ${ }^{7}$ provide both numerical and experimental studies of a new impedance-matching approach as applied to the cavity absorption problem. Again, a layer of sound-absorbing material is mounted a short distance away from an active wall. The creation of a pressure-release condition at the back surface of the layer ${ }^{1,2}$ is abandoned for the new impedance-matching condition, in which the reflected wave inside the cavity is to be minimized. The authors demonstrate the effectiveness of the approach both numerically and experimentally for normal incident waves of single frequency excitations. Numerical results showed absorption coefficients ranging from 0.8 to 1.0 could be achieved from 100 to $2000 \mathrm{~Hz}$.

Further study of the impedance-matching boundary condition for use in a hybrid sound absorption system is presented here. Previous work has dealt primarily with single frequency excitations and has provided no direct comparison between the pressure-release and impedance-matching conditions in terms of performance and control effort. In this experimental work, the performance and control effort of the active portions for the pressure-release and impedancematching boundary condition approaches will be compared in the $100-$ to $1000-\mathrm{Hz}$ broadband frequency range. Additional results will be presented which demonstrate the relative sensitivities of the two approaches to both the thickness of the absorbing layer and the depth of the cavity backing this layer.

A brief explanation of the motivation behind impedancematching shall be offered before continuing to a description of the experimental approach and associated results. The basis for the pressure-release condition stems from the desire to

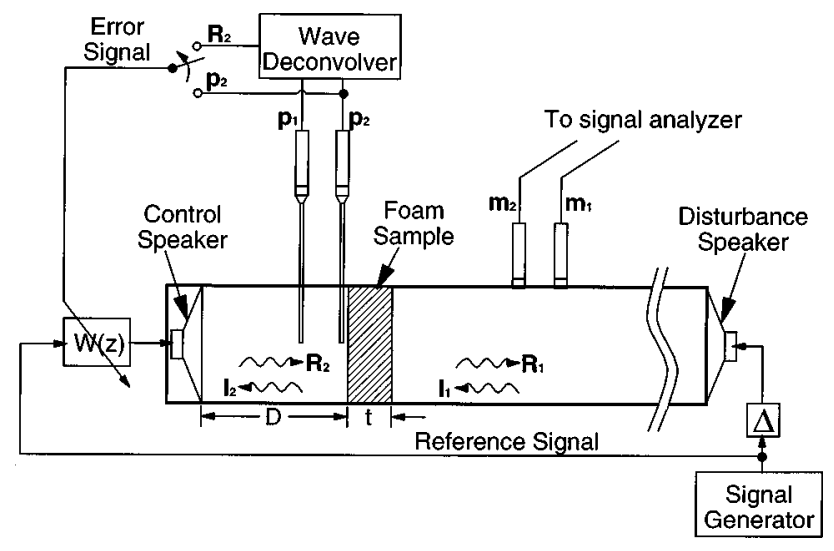

FIG. 2. Schematic of the hybrid absorption system inside a standing wave tube.

have maximum particle velocity occurring in the foam, thereby maximizing viscous losses in the incident pressure wave. This method is limited because it has only a local effect, observed at the location where the pressure is minimized. With impedance matching, however, the active component modifies the system so as to mimic the ambient acoustic impedance at the surface of the absorbing layer, preventing reflection of the incident wave. Hence, the main benefit of the impedance-matching boundary condition is that, unlike the pressure-release boundary condition, the impedance-matching condition induces more than a localized effect.

\section{CONTROL ALGORITHM}

The active portion of the hybrid absorption system relies upon the application of the filtered-X LMS (least mean square) feedforward algorithm in a single-input, singleoutput (SISO) configuration. Feedforward algorithms of this type have been successfully employed in various vibration and noise control scenarios including Active Structural Acoustic Control (ASAC) and Active Noise Control (ANC). ${ }^{8,9}$

A block diagram for the SISO control system is given as Fig. 1. Feedforward control relies heavily upon the determination of a reference signal, $x(k)$, which is coherent with the disturbance, $d(k)$, and independent of the control signal, $u(k)$. The subscript $k$ indicates the $k$ th time sample. In many applications it is possible to measure the reference signal directly, while in others electronic compensation is necessary to prevent feedback of the control signal into the measured reference signal. Once determined, the reference signal is filtered via an adaptive finite impulse response (FIR) filter, $W(z)$, to produce the control signal.

The adaptive filter, or compensator, consists of a finite number of coefficients that are updated with each time step of the algorithm so as to minimize a defined error signal. The sample rate and convergence parameter dictate the speed with which the filter coefficients converge. A FIR filter representation of the control path transfer function, $T_{c e}$, is required before the convergence process may be initiated. This model, $\widetilde{T}_{c e}$, is either measured or computed, and is used to filter the reference signal to create the filtered-X signal, $\tilde{x}_{k}$. 
The LMS algorithm then combines knowledge of the error signal and the filtered- $X$ signal to compute the updated compensator coefficients at each time step. For a thorough explanation of the LMS algorithm in the context of a multiple channel control system the reader is referred to the work of Elliot et al. ${ }^{10}$ In practice, the FIR filter modeling the control transfer function is determined by a system identification procedure that uses an algorithm similar to that of the control algorithm itself. ${ }^{11}$

A Texas Instruments TMS320C30 digital signal processing (DSP) board was used to implement the control algorithm. The board was resident in an Intel 80386 PC host computer and the controler codes were written in " $\mathrm{C}$ " language with embedded assembly code instructions. The code allows for user definition of the filter lengths, sample rate, and convergence parameter. Another feature of the control code is the ability to delay the disturbance signal by a number of time samples to ensure adequate response time for the controler and avoid causality issues.

\section{EXPERIMENTAL SETUP}

The tests were conducted inside a cylindrical standing wave (or impedance) tube having a diameter of $10 \mathrm{~cm}$ and a length of $1.2 \mathrm{~m}$. The tube has lower and upper frequency limits of 90 and $2000 \mathrm{~Hz}$, respectively. A depiction of the setup is given in Fig. 2. A white noise generator was used to create a broadband disturbance signal with uniform frequency content from 0 to $1600 \mathrm{~Hz}$. This signal is issued first to the controler as a reference signal and second to the disturbance speaker with a variable delay. The disturbance speaker is mounted at one the end of the wave tube, while the control speaker is mounted at the opposite end, behind the absorbing layer and the air cavity. Inside the cavity, two microphones, labeled $p_{1}$ and $p_{2}$, are shown in Fig. 2. The $p_{2}$ microphone is used as the error sensor for configurations with the pressure-release boundary condition. For the impedance-matching boundary condition, the error signal to be minimized is the reflected wave in the cavity $R_{2}$, which is measured by means of an analog wave deconvolution circuit in conjunction with the two cavity microphones. The deconvolution circuit follows a pattern initially presented by Fahy. ${ }^{12}$ The two microphones, labeled $m_{1}$ and $m_{2}$, outside the cavity are used for computation of the absorptivity using the ASTM two-microphone technique. ${ }^{13}$

TABLE I. Comparison of average absorption coefficient for impedancematching and pressure-release boundary conditions for various system configurations (100 to $1000 \mathrm{~Hz})$.

\begin{tabular}{cccccc}
\hline \hline \multirow{2}{*}{$\begin{array}{c}\text { Cavity depth } \\
(\mathrm{mm})\end{array}$} & \multicolumn{5}{c}{ Foam thickness (mm) } \\
\cline { 2 - 5 } & 12.7 & 25.4 & 38.1 & 50.8 & B.C. \\
\hline 60 & 0.96 & 0.95 & 0.90 & 0.87 & I.M. \\
& 0.79 & 0.89 & 0.85 & 0.85 & P.R. \\
80 & 0.95 & 0.94 & 0.87 & 0.86 & I.M. \\
& 0.76 & 0.87 & 0.83 & 0.82 & P.R. \\
100 & 0.91 & 0.91 & 0.85 & 0.83 & I.M. \\
& 0.72 & 0.82 & 0.80 & 0.81 & P.R. \\
\multirow{2}{*}{120} & 0.88 & 0.87 & 0.81 & $\cdots$ & I.M. \\
& 0.67 & 0.78 & 0.76 & $\cdots$ & P.M. \\
\hline \hline
\end{tabular}

The method used for computation of the absorption properties has frequency limitations on the low end dictated by the phase mismatch of the measurement microphones, and on the high end by the microphone spacing used. These limitations are well defined in Ref. 13 and resulted in a usable frequency range from approximately 100 to $1600 \mathrm{~Hz}$ in these experiments.

The sensitivity of the hybrid absorption system is to be studied relative to the cavity depth, $D$, and the thickness of the foam sample, $t$ (see Fig. 2). Tests were performed for cavity depths of $60,80,100$, and $120 \mathrm{~mm}$ and foam thicknesses of 12.7, 25.4, 38.1, and $50.8 \mathrm{~mm}$. For each configuration, the controller ran the filtered-X LMS adaptive feedforward control algorithm with a sample rate of $3200 \mathrm{~Hz}$, using a 140-coefficient FIR compensator and a 40-sample delay between the reference and disturbance signals. The 40sample delay was introduced to remove any causality constraints from the experiment. The issue of causality in feedforward broadband applications has been addressed by Burdisso et al. ${ }^{14}$ In practical applications, the need for a coherent, a priori reference signal may limit the implementation and will require some strategy based on the particular configuration.

\section{EXPERIMENTAL RESULTS}

Experiments using both the pressure-release and impedance-matching hybrid absorption systems shall be described in this section. Results contrasting the performance of the impedance-matching and pressure-release boundary conditions shall first be presented. Following this discussion, the sensitivity of the hybrid absorption system to change in the cavity depth and the foam thickness will be quantified for each boundary condition.

\section{A. Performance comparison}

For each boundary condition (pressure release and impedance matching), 15 cavity configurations were studied in this work, in which both the cavity depth and foam thickness were independently varied. A spectrum yielding the absorption coefficient as a function of frequency was measured for each configuration. The FIR compensator coefficients of the controller were also recorded for each case. From these coefficients the frequency response function of the controller may be obtained, providing a quantifier of the controller effort for direct comparison of any two configurations. Rather than attempting the cumbersome task of presenting all of this data, another means for comparing configurations shall be contrived. First, the absorption coefficient spectrum shall be replaced by the linear average of the absorption coefficient. Since our concern lies in the frequency range between 100 and $1000 \mathrm{~Hz}$, the average reflects only this portion of the spectrum. As a reminder, it is noted that the wave tube has a lower frequency limit of $90 \mathrm{~Hz}$, preventing comparison of the two approaches below roughly $100 \mathrm{~Hz}$. Second, in place of the control compensator frequency response the average compensator gain over the $100-$ to $1000-\mathrm{Hz}$ frequency range shall be presented. After considering this abbreviated format of the experimental data, the results for two configurations shall be described in detail. 
TABLE II. Average control compensator gain (dB) for pressure-release and impedance-matching boundary conditions for various system configurations (100 to $1000 \mathrm{~Hz})$.

\begin{tabular}{cccccc}
\hline \hline \multirow{2}{*}{$\begin{array}{c}\text { Cavity depth } \\
(\mathrm{mm})\end{array}$} & 12.7 & 25.4 & 38.1 & 50.8 & B.C. \\
\cline { 2 - 5 } & -6.62 & -7.57 & -9.33 & -9.58 & I.M. \\
\hline \multirow{2}{*}{60} & -2.54 & -4.40 & -6.11 & -7.19 & P.R. \\
& -6.98 & -8.18 & -9.22 & -11.09 & I.M. \\
80 & -3.89 & -5.42 & -6.49 & -7.91 & P.R. \\
& -7.70 & -9.21 & -9.90 & -11.00 & I.M. \\
100 & -3.65 & -6.11 & -6.71 & -8.75 & P.R. \\
& -7.97 & -9.48 & -10.55 & $\ldots$ & I.M. \\
\multirow{2}{*}{120} & -4.62 & -6.56 & -7.80 & $\ldots$ & P.R. \\
\hline \hline
\end{tabular}

Tables I and II, respectively, reflect the performance and controller effort quantifiers described in the preceding paragraph. As shown in Table I, the average absorption coefficient over the $100-$ to $1000-\mathrm{Hz}$ range is higher with impedance matching than with pressure release in each of the 15 cases. The average absorption coefficient was computed as a linear average of the absorption coefficients over the frequency range in order to give a single quantitative value for the absorption effects of each case. The results in Tables I and II are not to say that impedance-matching provides greater absorption over the entire frequency range in every case, but only that the average absorption is higher. In some instances the pressure-release condition provided better absorption coefficients over certain frequency ranges. From Table II, it is evident that the impedance-matching approach requires less control effort, in all instances yielding an average compensator gain more than $3 \mathrm{~dB}$ less than the corresponding pressure-release configuration. Note that a $3-\mathrm{dB}$ difference in the compensator gain corresponds to approximately a factor of 2 in terms of power consumption.

Having in mind an overview of the results, a more thorough description of two specific configurations shall now be given. First, let us consider the upper-leftmost configuration presented in the tables, corresponding to a cavity depth of 60

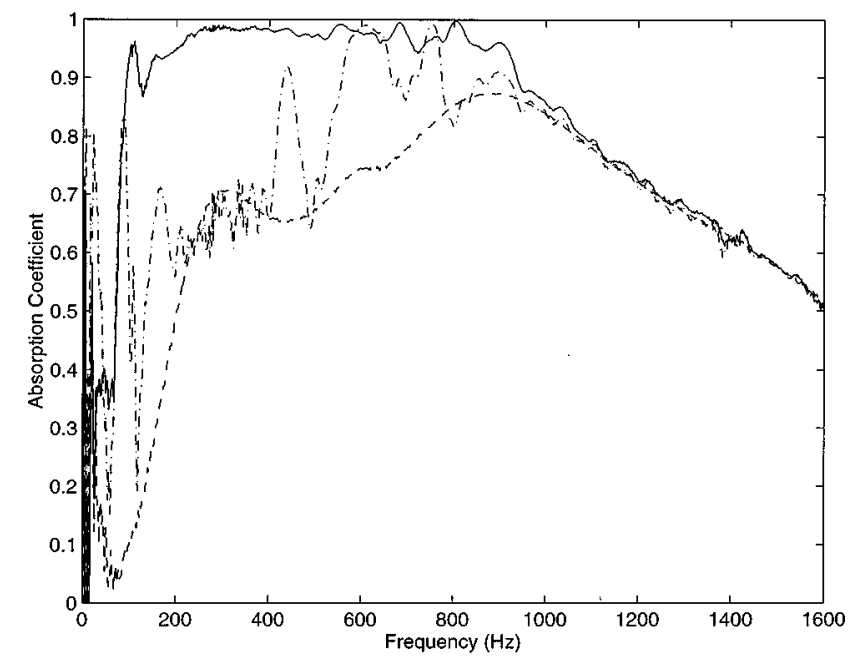

FIG. 3. Absorption coefficient frequency spectrum for $D=60 \mathrm{~mm}$ and $t$ $=12.7 \mathrm{~mm}$. Condition: — impedance matching, -..- pressure release, --- passive.

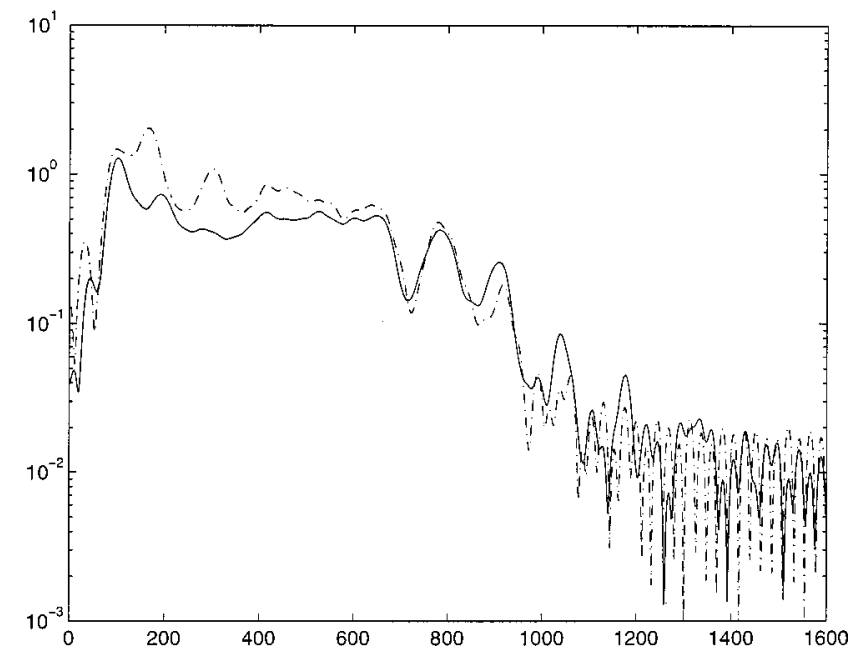

FIG. 4. Control compensator magnitude (V/V) for $D=60 \mathrm{~mm}$ and $t$ $=12.7 \mathrm{~mm}$. Condition: — impedance matching, $-\cdots-\cdot$ - pressure release.

$\mathrm{mm}$ and a foam thickness of $12.7 \mathrm{~mm}$. The measured absorption coefficient is given as a function of frequency in Fig. 3. Again, note that below about $100 \mathrm{~Hz}$ the measurement of the absorption coefficient is no longer accurate. Nearly perfect absorption is achieved from 100 to $1000 \mathrm{~Hz}$ with the impedance-matching boundary configuration and throughout this range the impedance-matching approach improves the absorption relative to the passive system alone. The pressurerelease approach, on the other hand, increases the absorption over the passive case for three considerably smaller frequency ranges and actually acts to lessen the absorption of the cavity for some frequencies. Table I indicates that the average absorption coefficient for this configuration is 0.17 greater with the impedance-matching condition. The magnitude of the compensator frequency response function is given in Fig. 4 for each boundary condition. It is apparent that the impedance-matching boundary condition requires less effort from the active component, especially between about 100 and $700 \mathrm{~Hz}$. Based on the average compensator gain data in Table II the pressure-release approach requires 2.5 times the power of the corresponding impedance-

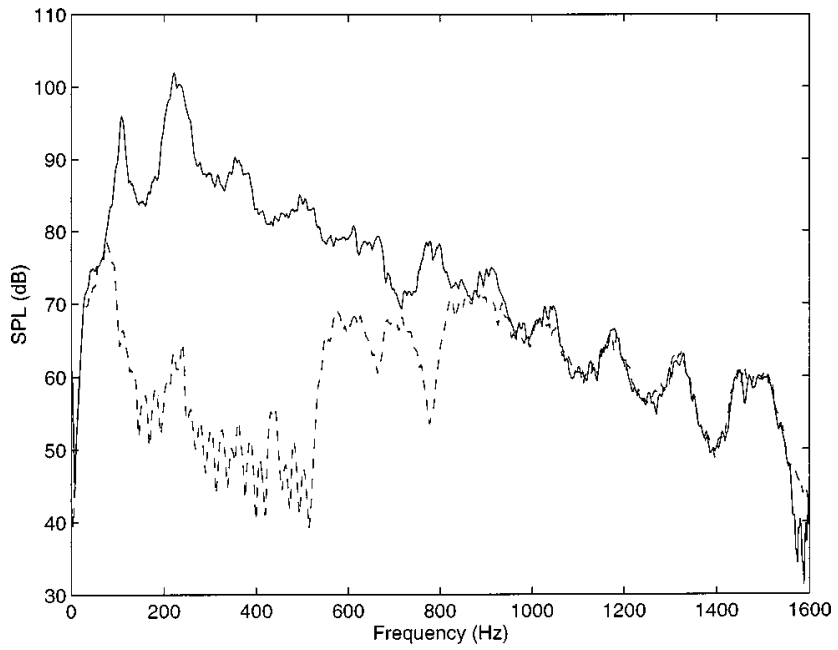

FIG. 5. Frequency spectrum of the impedance-matching error signal: - before control, --- after control. 


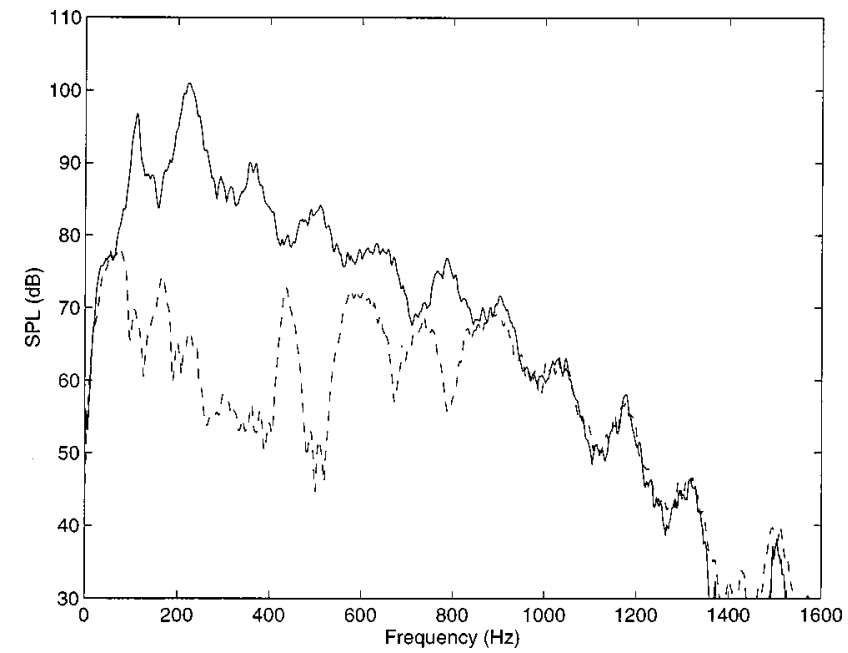

FIG. 6. Frequency spectrum of the pressure-release error signal: before control, --- after control.

matching system. Finally, Figs. 5 and 6 depict the error signal frequency responses for the impedance-matching and pressure-release conditions, respectively. Over the 100- to $1000-\mathrm{Hz}$ frequency range an overall reduction of $20.8 \mathrm{~dB}$ was observed for the impedance-matching case, while a 19.8-dB reduction was achieved for the pressure-release configuration. The similarity in overall reduction of the error signals assures independence of the hybrid absorption system relative to the controler, isolating the effects of each boundary condition on the performance.

As a second in-depth comparison of the two hybrid absorption approaches we shall consider a cavity depth of 100 $\mathrm{mm}$ and a foam thickness of $50.8 \mathrm{~mm}$, corresponding to the lower right of the tables presented earlier in this work. In Fig. 7, the measured absorption coefficient as a function of frequency is given. Here we see that both boundary conditions result in a degradation of absorption between 300 and $700 \mathrm{~Hz}$. While the impedance-matching condition provides better absorption in this range, the pressure-release condition yields greater absorption from 100 to $300 \mathrm{~Hz}$. Referring to

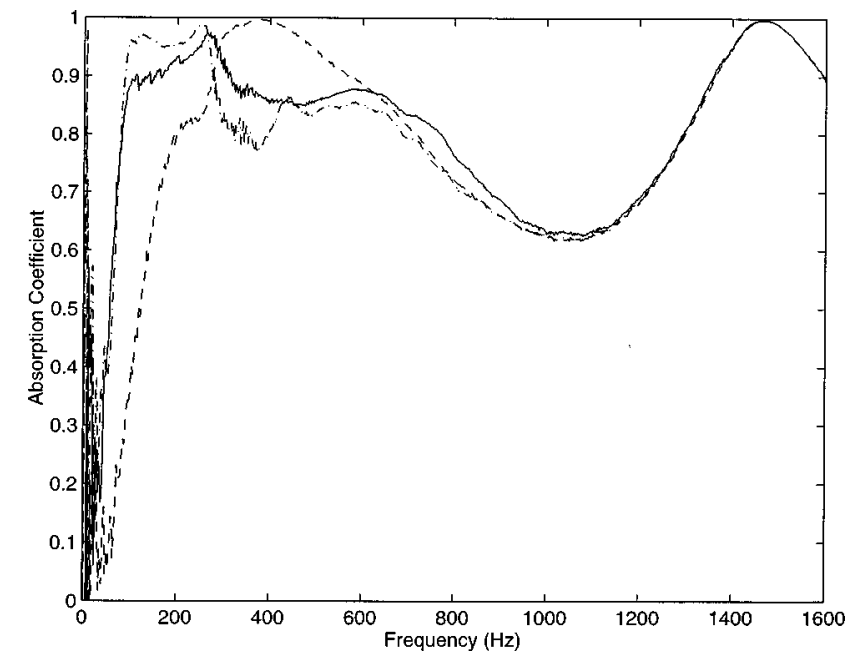

FIG. 7. Absorption coefficient frequency spectrum for $D=100 \mathrm{~mm}$ and $t$ $=50.8 \mathrm{~mm}$. Condition: — impedance matching, -..- pressure release, --- passive.

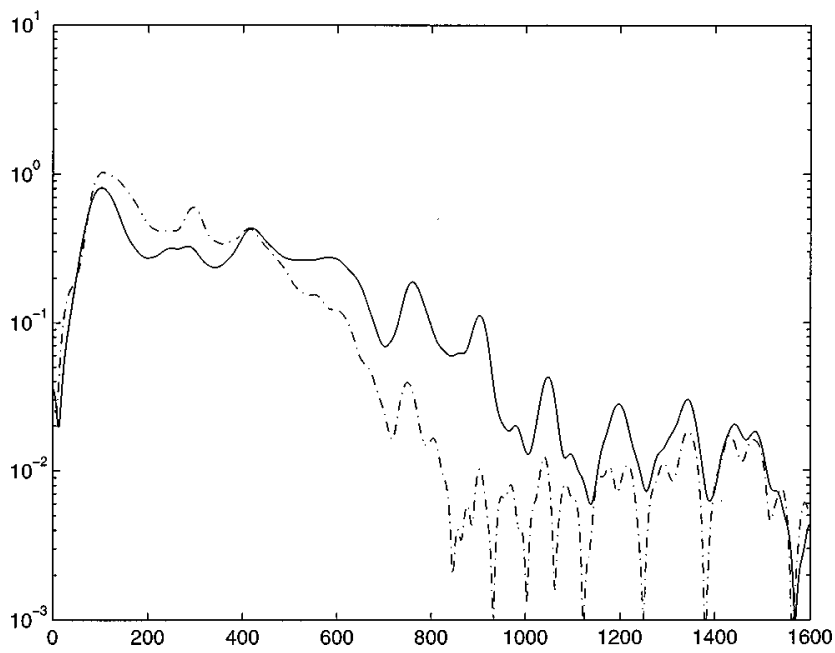

FIG. 8. Control compensator magnitude (V/V) for $D=100 \mathrm{~mm}$ and $t$ $=50.8 \mathrm{~mm}$. Condition: — impedance matching, $\cdots \cdots$ pressure release.

Table I, we find a difference of only 0.01 in the average absorption coefficient for these two configurations. As with the previous case, however, the required controller effort is significantly less for the impedance-matching case, as the pressure-release approach requires $50 \%$ more power. This is evidenced by Fig. 8, which contains the compensator magnitude frequency responses determined for each boundary condition with this cavity configuration.

\section{B. Sensitivity analysis}

In the preceding section the impedance-matching boundary condition was compared to the pressure-release boundary condition both in terms of absorption performance and control effort. Here we seek to demonstrate the sensitivity of each approach to changes in the cavity depth and the thickness of the absorbing foam layer. As a first indicator Tables I and II may again be consulted. First, let us consider the impedance-matching data. It is easily seen that as the foam layer is thickened, the average absorption coefficient and the average compensator gain each decrease. A similar trend is

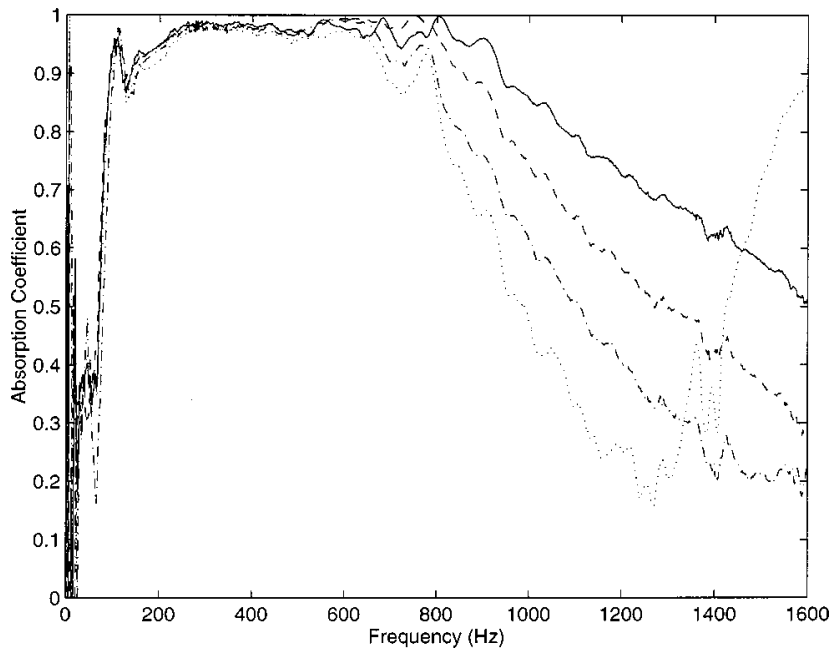

FIG. 9. Absorption coefficient frequency spectrum with impedance matching for $t=12.7 \mathrm{~mm} . D$ : $60 \mathrm{~mm}, \cdots-\cdots 0 \mathrm{~mm},---100 \mathrm{~mm}, \cdots 120$ $\mathrm{mm}$. 


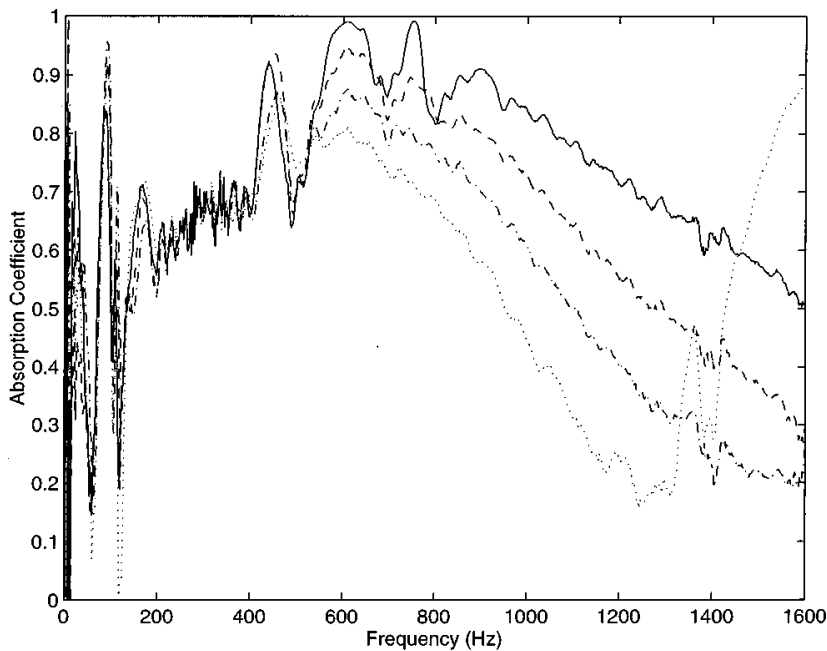

FIG. 10. Absorption coefficient frequency spectrum with pressure release for $t=12.7 \mathrm{~mm}$. $D:-60 \mathrm{~mm}, \cdots 80 \mathrm{~mm},---100 \mathrm{~mm}, \cdots 120 \mathrm{~mm}$.

observed as the cavity depth is increased. Second, we consider the results observed with the pressure-release boundary condition. With increasing foam thickness, a decrease is again observed in the average compensator gain; however, the average absorption coefficient is seen to increase and then decrease, suggesting the presence of an optimal thickness. This optimal thickness likely depends on the absorbing material, the cavity depth, and the frequency range of interest. As the cavity depth is increased, a general decline is observed in both the average absorption coefficient and the average compensator gain. The performance of the impedance-matching condition is seen to be less sensitive to the cavity depth than the pressure-release condition. Physical insight into these trends would require an analytical model which includes the physical properties of the absorption material.

The average absorption coefficient provides a useful glance at the sensitivity of the hybrid absorption system to the cavity geometry, but it does not offer a means by which to study this sensitivity. In order to understand better the

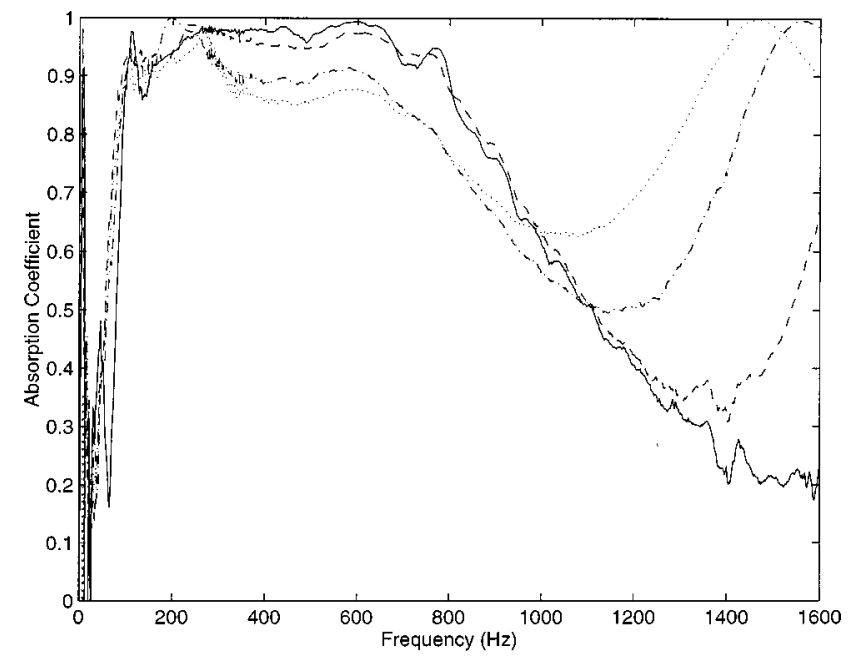

FIG. 11. Absorption coefficient frequency spectrum with impedance matching for $D=100 \mathrm{~mm}$. $t:-12.7 \mathrm{~mm},-\cdots-25.4 \mathrm{~mm},--38.1 \mathrm{~mm}, \cdots$ $50.8 \mathrm{~mm}$.

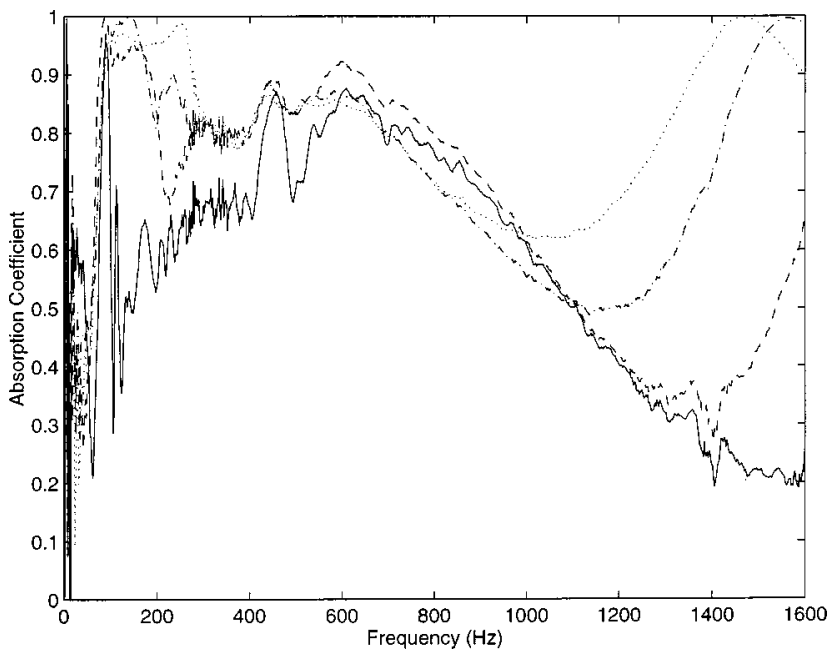

FIG. 12. Absorption coefficient frequency spectrum with pressure release for $D=100 \mathrm{~mm}$. $t:-12.7 \mathrm{~mm}, \cdots 25.4 \mathrm{~mm},---38.1 \mathrm{~mm}, \cdots 50.8$ $\mathrm{mm}$.

effect of the cavity geometry on the system, the frequency dependence of the absorption coefficient for varied cavity geometries shall be considered. Figure 9 contains the absorption coefficient frequency spectra measured with the impedance-matching approach, a fixed foam thickness of $12.7 \mathrm{~mm}$, and four cavity depths. A rolloff in the absorption coefficient is observed near $700 \mathrm{~Hz}$, the grade of this decline becoming steeper with increasing cavity depths. This is likely a passive effect stemming from a change in the cavity resonance as the depth of the cavity is changed. Figure 10 represents the corresponding results for the pressure-release condition, depicting a nearly identical trend.

Lastly, the frequency dependence of the absorption coefficient shall be investigated under a fixed cavity depth and varied foam thickness for each boundary condition. The cavity depth used for this study was $100 \mathrm{~mm}$. In Fig. 11 the sensitivity of the impedance-matching approach to a varied foam thickness is illustrated. Above about $1000 \mathrm{~Hz}$ the increasing foam thickness results in an increase in the absorptivity, which is again suspected to be caused by a shifting of the cavity resonance. Between 300 and $1000 \mathrm{~Hz}$ the increased foam thickness results in a deterioration of the absorption coefficient. The high-frequency absorption behavior observed with impedance matching is mirrored in the results obtained with pressure release, as depicted in Fig. 12. This similarity strengthens the conclusion that this phenomenon is a passive effect as it is independent of the chosen boundary condition. In the low-frequency range, between about 100 and $800 \mathrm{~Hz}$, the pressure-release approach yields an irregular variation in the absorption coefficient with respect to the foam thickness. It is evident that the pressure-release approach is more sensitive to this change than the impedancematching approach.

\section{CONCLUSIONS}

An experimental study was conducted which demonstrates the superiority of the impedance-matching technique over that of the pressure-release approach for application to the active component of a broadband hybrid absorption 
system comprised of an active wall positioned at the rear of an air cavity terminated by a sound-absorbing layer. Sensing and minimizing the reflected wave inside the cavity achieved greater absorption coefficients than by achieving a pressurerelease condition at the back of the absorbing layer. Moreover, this increased performance was obtained with substantially less control effort, in many cases requiring half the power of the corresponding system based upon the pressurerelease approach. Furthermore, with the impedance-matching approach absorption coefficients between 0.8 and 1.0 were generally attained over the $100-1000-\mathrm{Hz}$ range, with specific geometries yielding absorption coefficients upwards of 0.9 throughout this frequency range. These results are expected to be applicable to other types of porous materials.

${ }^{1}$ D. Guicking and E. Lorenz, "An Active Sound Absorber with Porous Plate,' Journal of Vibration, Acoustics, Stress and Reliability in Design 106, 389-392 (1984).

${ }^{2}$ D. Thenail, M. Galland, M. Sunyach, and M. Sunhack, "Active Enhancement of the Absorbent Properties of a Porous Material," Smart Mater. Struct. 3, 18-25 (1994).

${ }^{3}$ M. Furstoss, D. Thenail, and M. A. Galland, "Surface Impedance Control for Sound Absorption: Direct and Hybrid Passive/Active Strategies,' J. Sound Vib. 203, 219-236 (1997).

${ }^{4}$ J. S. Bolton and E. R. Green, "Smart foams for active absorption of sound,' in Second Conference on Recent Advances in Active Noise Con- trol of Sound and Vibration, Blacksburg, VA (1993), pp. 139-149.

${ }^{5}$ C. R. Fuller, M. J. Bronzel, C. H. Gentry, and D. E. Whittington, 'Control of Sound Radiation/Reflection with Adaptive Foams,' in Proceedings of Noise-Con 1994 (1994), pp. 429-436.

${ }^{6}$ I. L. Ver and M. Digman, "Active Liner System for Jet Engine Exhaust Silencers,' Internoise 95, Newport Beach, CA (1995), pp. 481-484.

${ }^{7}$ S. Beyene and R. A. Burdisso, "A New Hybrid Passive/Active Noise Absorption System,' J. Acoust. Soc. Am. 101, 1512-1515 (1997).

${ }^{8}$ J. P. Smith, C. R. Fuller, and R. A. Burdisso, "Control of Broadband Radiated Sound with Adaptive Structures,' Proc. SPIE Conf., Smart Structures and Intelligent Systems, 1917 (Pt. 1), 587-597 (February 1993).

${ }^{9}$ S. J. Elliott, P. A. Nelson, I. M. Stothers, and C. C. Boucher, "In Flight Experiments on the Active Control of Propeller-Induced Cabin Noise,' J. Sound Vib. 140, 219-238 (1990).

${ }^{10}$ S. J. Elliott, I. M. Stothers, and P. A. Nelson, "A Multiple Error LMS Algorithm and its application to the Active Control of Sound and Vibration,' IEEE Trans. Acoust., Speech, Signal Process. 35, 1423-1434 (1987).

${ }^{11}$ B. Widrow and S. D. Stearns, Adaptive Signal Processing (Prentice-Hall, Englewood Cliffs, NJ, 1985).

${ }^{12}$ F. J. Fahy, "A Technique for Measuring Sound Intensity with a Sound Level Meter,' Noise Control Eng. 9, 155-162 (1977).

13 “'Standard Test Method for Impedance and Absorption of Acoustical Materials by the Impedance Tube Method,' ASTM C384, Philadelphia (1990).

${ }^{14}$ R. A. Burdisso, J. S. Vipperman, and C. R. Fuller, "Causality analysis of feedforward-controlled systems with broadband inputs,' J. Acoust Soc. Am. 94, 234-242 (1993). 